\title{
Dynamical Behavior of a Novel Impulsive Switching Model for HLB with Seasonal Fluctuations
}

\author{
Shujing Gao $\mathbb{D}^{1,2}$ Lei Luo, ${ }^{1}$ Shuixian Yan, ${ }^{1}$ and Xinzhu Meng $\mathbb{D}^{3}$ \\ ${ }^{1}$ Key Laboratory of Jiangxi Province for Numerical Simulation and Emulation Techniques, Gannan Normal University, \\ Ganzhou 341000, China \\ ${ }^{2}$ National Research Center of Navel Orange Engineering and Technology, Gannan Normal University, Ganzhou 341000, China \\ ${ }^{3}$ College of Mathematics and Systems Science, Shandong University of Science and Technology, Qingdao 266590, China
}

Correspondence should be addressed to Shujing Gao; gaosjmath@126.com

Received 16 November 2017; Accepted 31 May 2018; Published 26 July 2018

Academic Editor: Alejandro Vargas

Copyright (c) 2018 Shujing Gao et al. This is an open access article distributed under the Creative Commons Attribution License, which permits unrestricted use, distribution, and reproduction in any medium, provided the original work is properly cited.

\begin{abstract}
This paper studies a new model for Huanglongbing with seasonal fluctuations. Switching coefficients and switching control schemes are considered in this model. The main purpose of this paper is to study the effects of switching control schemes on dynamics of the model. Firstly, we theoretically investigate the basic reproductive number and its computation formulae for general impulsive switching model with periodic environment. Secondly, the basic reproductive number and global dynamics of the impulsive switching model for Huanglongbing are analyzed. Finally, numerical results indicate that spring and autumn are the optimum seasons for killing psyllids, and winter is the optimum season for removing infected trees.
\end{abstract}

\section{Introduction}

Citrus Huanglongbing (HLB), also known as citrus greening, is one of the most devastating diseases of citrus worldwide [1]. The Asiatic citrus psyllid and Diaphorina citri Kuwayama are the only two known vectors of the debilitating citrus HLB [2]. Nearly 50 countries are affected by this disease especially in Asian, African, and American countries, such as Brazil, USA, and China. It was estimated by the University of Florida in 2012 that, in Florida, HLB had resulted in the loss of 6611 jobs from 2006 throughout 2011, 1.3 billion in revenue to growers, and 3.63 billion in economic activity [3]. In São Paulo, $64.1 \%$ of the commercial citrus blocks and $6.9 \%$ of the citrus trees were affected by HLB in 2012 [4]. Till now, in China, the damaged area of citrus is more than $80 \%$ of the total cultivated area [5]. Unfortunately, there currently is no cure for HLB nor is there any naturally occurring citrus cultivar that is resistant to HLB.

HLB is a vector-transmitted bacterial infection through psyllids [6]. Since the pioneering work of MacDonald and Barbour on schistosomiasis [7, 8], many mathematical models have been proposed in analyzing the spread and control of vector-borne diseases, such as malaria, dengue fever, schistosomiasis, West Nile disease, HLB (see [9-14] and references therein). In [8], Barbour formulated a mathematical model of schistosomiasis as follows:

$$
\begin{aligned}
& \frac{d I_{h}(t)}{d t}=a S_{h} I_{v}-\mu_{1} I_{h}, \\
& \frac{d I_{v}(t)}{d t}=b S_{v} I_{h}-\mu_{2} I_{v}, \\
& \frac{d S_{h}(t)}{d t}=\mu_{1}-a S_{h} I_{v}-\mu_{1} S_{h}, \\
& \frac{d S_{v}(t)}{d t}=\mu_{2}-b S_{v} I_{h}-\mu_{2} S_{v},
\end{aligned}
$$

where $S_{h}\left(I_{h}\right)$ is the susceptible (infected) host population, $S_{v}\left(I_{v}\right)$ is the susceptible (infected) vector population, $a$ and $b$ are infection rates, $\mu_{1}\left(\mu_{2}\right)$ is the natural mortality rate of the host (vector) population.

As we know, young flush (initial infection of newly developing cluster of young leaves) become infectious within 15 days after receiving an inoculum of bacteria [15], and 
symptoms of HLB do not appear on leaves for months to years after initial infection. The survey results from $[16,17]$ indicated that the incubation period from grafting to development of HLB symptoms is 3 to 12 months under greenhouse conditions. For large trees in a field situation, the incubation period may be much longer, up to more than 5 years. This means that HLB has a long incubation period during which the plant is asymptomatic but infectious [18]. Therefore, in this paper, we classify the citrus tree into four compartments: susceptible $S_{h}$, infected and asymptomatic but not yet infectious $E_{h}$, infectious and asymptomatic $I_{1}$, and infectious and symptomatic $I_{2}$, and the psyllids vector into two compartments: susceptible class $S_{v}$ and infected class $I_{v}$. Let $N_{h}(t)$ and $N_{v}(t)$ be the total numbers of citrus trees and psyllids, respectively, at time $t$ in a grove. That is, $N_{h}(t)=S_{h}(t)+E_{h}(t)+I_{1}(t)+I_{2}(t)$ and $N_{v}(t)=I_{v}(t)+$ $S_{v}(t)$. Assume that removed trees are immediately replaced by susceptible trees, keeping the grove size constant [19]. Thus, $N_{h}(t)$ is constant and denotes $N_{h}$. Inspired by the idea of Barbour's model (1), considering HLB transmission between citrus trees and psyllids, we establish the following HLB model.

$$
\begin{aligned}
& \frac{d E_{h}(t)}{d t}=\frac{a S_{h} I_{v}}{N_{h}}-\mu_{1} E_{h}-\alpha E_{h}-d E_{h}, \\
& \frac{d I_{1}(t)}{d t}=\alpha E_{h}-\mu_{1} I_{1}-\theta I_{1}-d I_{1}, \\
& \frac{d I_{2}(t)}{d t}=\theta I_{1}-\mu_{1} I_{2}-d I_{2}, \\
& \frac{d I_{v}(t)}{d t}=\frac{c S_{v} I_{1}}{N_{h}}+\frac{b S_{v} I_{2}}{N_{h}}-\mu_{2} I_{v}, \\
& \frac{d S_{h}(t)}{d t}=\mu_{1} N_{h}-\mu_{1} S_{h}-\frac{a S_{h} I_{v}}{N_{h}}+d E_{h}+d I_{1}+d I_{2}, \\
& \frac{d S_{v}(t)}{d t}=\Lambda-\frac{c S_{v} I_{1}}{N_{h}}-\frac{b S_{v} I_{2}}{N_{h}}-\mu_{2} S_{v},
\end{aligned}
$$

where $\alpha$ and $\theta$ are the conversion rates, $\mu_{1}\left(\mu_{2}\right)$ is the natural mortality rate of the citrus tree (psyllids), $a$ is the infection rate from infected psyllids to susceptible trees, $b$ is the infection rate from infectious and symptomatic trees to psyllids, $c=k b$ means the infection rate from infectious and asymptomatic trees to psyllids, and $k(0<k \leq 1)$ is the proportional coefficient, $d$ is the mortality rate of citrus trees due to illness, and $\Lambda$ is the constant recruitment rate of psyllids.

In general, spraying insecticides over entire groves as well as eliminating infected symptomatic trees have always been implemented in controlling the spread of HLB. In Thailand, 3-6 sprays per year was required during flush periods to rehabilitate citrus production in a HLB-infected area [20]. However, the common assumption about the continuity of control activities is contradictory from the reality that the control behavior usually occurs in regular pulses [21]. Spraying insecticides is generally applied at a fixed time, and the effect of pesticide spraying depends on the time of initial spraying and frequency. By considering impulsive control strategies, system (2) can be described by impulsive differential equations as follows:

$$
\begin{aligned}
\frac{d E_{h}(t)}{d t} & =\frac{a S_{h} I_{v}}{N_{h}}-\mu_{1} E_{h}-\alpha E_{h}-d E_{h}, \\
\frac{d I_{1}(t)}{d t} & =\alpha E_{h}-\mu_{1} I_{1}-\theta I_{1}-d I_{1}, \\
\frac{d I_{2}(t)}{d t} & =\theta I_{1}-\mu_{1} I_{2}-d I_{2}-\gamma I_{2}, \\
\frac{d I_{v}(t)}{d t} & =\frac{c S_{v} I_{1}}{N_{h}}+\frac{b S_{v} I_{2}}{N_{h}}-\mu_{2} I_{v}, \\
\frac{d S_{h}(t)}{d t} & =\mu_{1} N_{h}-\mu_{1} S_{h}-\frac{a S_{h} I_{v}}{N_{h}}+d E_{h}+d I_{1}+d I_{2}+\gamma I_{2}, \\
\frac{d S_{v}(t)}{d t} & =\Lambda-\frac{c S_{v} I_{1}}{N_{h}}-\frac{b S_{v} I_{2}}{N_{h}}-\mu_{2} S_{v} . \\
t & \in\left(t_{k-1}, t_{k}\right], \\
I_{v}\left(t^{+}\right) & =(1-p) I_{v}(t), \\
S_{v}\left(t^{+}\right) & =(1-p) S_{v}(t), \\
t & =t_{k} .
\end{aligned}
$$

where $\gamma$ is the removal rate of infected symptomatic trees and $p$ is the killing rate of psyllids by insecticide spraying.

Furthermore, in endemic areas, removing of citrus trees is always based predominantly on the presence of visible symptoms [22]. All of the trees showing HLB symptoms should be removed 3 times in each year [20]. These imply that the infection rates and the removal rate vary with season fluctuations. Thus, it is necessary to consider that some coefficients of model (3) are time-varying and switching in time. Suppose that some parameters are modeled as switching parameters and governed by a switching rule $\sigma(t):\left(t_{k-1}, t_{k}\right]$ $\rightarrow\{1,2, \ldots, m\}=\mathscr{P}, k=1,2, \ldots$, where $m$ is the number of the subsystems and $\sigma(t)$ is a piecewise continuous switching rule such that $\sigma(t)=i_{k} \in \mathscr{P}$ for all $t \in\left(t_{k-1}, t_{k}\right]$. The switching times $\left\{t_{k}\right\}$ satisfy $t_{k+1}>t_{k}>0$ and $\lim _{k \rightarrow \infty} t_{k}=\infty$. Define the set of all switching rules by $\mathscr{I}$. Motivated by above fact, we yield the switching HLB model with impulsive control:

$$
\begin{aligned}
& \frac{d E_{h}(t)}{d t}=\frac{a_{\sigma} S_{h} I_{v}}{N_{h}}-\mu_{1} E_{h}-\alpha E_{h}-d E_{h}, \\
& \frac{d I_{1}(t)}{d t}=\alpha E_{h}-\mu_{1} I_{1}-\theta I_{1}-d I_{1}, \\
& \frac{d I_{2}(t)}{d t}=\theta I_{1}-\mu_{1} I_{2}-d I_{2}-\gamma_{\sigma} I_{2} \\
& \frac{d I_{v}(t)}{d t}=\frac{c_{\sigma} S_{v} I_{1}}{N_{h}}+\frac{b_{\sigma} S_{v} I_{2}}{N_{h}}-\mu_{2} I_{v}, \\
& \frac{d S_{h}(t)}{d t}=\mu_{1} N_{h}-\mu_{1} S_{h}-\frac{a_{\sigma} S_{h} I_{v}}{N_{h}}+d E_{h}+d I_{1}+d I_{2}+\gamma_{\sigma} I_{2}, \\
& \frac{d S_{v}(t)}{d t}=\Lambda-\frac{c_{\sigma} S_{v} I_{1}}{N_{h}}-\frac{b_{\sigma} S_{v} I_{2}}{N_{h}}-\mu_{2} S_{v},
\end{aligned}
$$




$$
\begin{aligned}
t & \in\left(t_{k-1}, t_{k}\right], \\
I_{v}\left(t^{+}\right) & =\left(1-p_{\sigma}\right) I_{v}(t), \\
S_{v}\left(t^{+}\right) & =\left(1-p_{\sigma}\right) S_{v}(t), \\
t & =t_{k},
\end{aligned}
$$

where $p_{\sigma}\left(0 \leq p_{\sigma} \leq 1\right)$ are the killing rates of psyllids by insecticide spraying at time $t_{k}(k=1,2, \ldots)$. The initial conditions for system (4) satisfy $E_{h}\left(t_{0}^{+}\right)=E_{h}\left(t_{0}\right) \geq 0, I_{1}\left(t_{0}^{+}\right)=I_{1}\left(t_{0}\right) \geq 0$, $I_{2}\left(t_{0}^{+}\right)=I_{2}\left(t_{0}\right) \geq 0, \quad I_{v}\left(t_{0}^{+}\right)=I_{v}\left(t_{0}\right) \geq 0, \quad S_{h}\left(t_{0}^{+}\right)=S_{h}\left(t_{0}\right)>0$, and $S_{v}\left(t_{0}^{+}\right)=S_{v}\left(t_{0}\right)>0$. The model flow diagram is depicted in Figure 1.

The spread of infectious diseases is influenced by many factors, such as the behavior of the human population and the environment in which it spread [23]. Consequently, it is more realistic to consider the periodic switching rule. Following the idea of [23], we assume that the switching rule $\sigma$ satisfies $t_{k}-t_{k-1}=\omega_{k}$ with $\omega_{k+m}=\omega_{k}$, and then $\omega=\sum_{k=1}^{m} \omega_{k}$ is the period of switch $\sigma$. Assume that $a_{i_{k}}=a_{k}, b_{i_{k}}=b_{k}$, $c_{i_{k}}=c_{k}, m_{i_{k}}=m_{k}$, and $p_{i_{k}}=p_{k}$ for $t \in\left(t_{k-1}, t_{k}\right]$, and $a_{k+m}=$ $a_{k}, b_{k+m}=b_{k}, c_{k+m}=c_{k}, m_{k+m}=m_{k}$, and $p_{k+m}=p_{k}$. Define $\mathscr{J}_{p}$ as the set of periodic switching rule.

Note that models (2) and (3) can be considered as special cases of model (4). If the parameters of model (4) are constant and not switching in time, that is, there is only one independent subsystem $(m=1)$, then model (4) yields to model (3). Further, if control strategies are not in use, in the case where the killing rate of psyllids $(p)$ and the removal rate of infected symptomatic trees $(\gamma)$ are zero, then model (3) reduces to model (2). Our main purpose is to explore the effects of switching control schemes on the dynamics properties of model (4).

The rest of this paper is organized as follows: In Section 2, some basic notations and useful results are given. In Section 3 , the threshold condition and global asymptotic stability of the disease-free periodic solution of system (4) are studied. Furthermore, sufficient condition for persistence of the disease is derived. Numerical simulations are given in Section 4. Brief discussion and conclusion are presented in Section 5.

\section{Some Useful Results}

2.1. Some Useful Results for Linear Impulsive Switching System. Before investigating system (4), we will present some notations and state some results for linear impulsive switching system with periodic environment.

Define $\mathbb{R}_{+}=\{x \in \mathbb{R} \mid x \geq 0\}, \mathbb{R}_{+}^{n}=\left\{\left(x_{1}, \ldots, x_{n}\right) \in \mathbb{R}^{n} \mid x_{i}\right.$ $\geq 0, i=1,2, \ldots, n\}$. Let $r(B)$ be the spectral radius of matrix $B$.

Consider a linear impulsive switching differential system:

$$
\begin{aligned}
\dot{x}(t) & =A_{\sigma}(t) x(t), \quad t \in\left(t_{k-1}, t_{k}\right], \\
x\left(t_{k}^{+}\right) & =P_{\sigma} x\left(t_{k}\right), \quad t=t_{k}, k \in \mathbb{N}, \\
x\left(t_{0}^{+}\right) & =x_{0}, \quad t_{0} \geq 0,
\end{aligned}
$$

where $x=\left(x_{1}, x_{2}, \ldots, x_{n}\right)^{T}, A_{\sigma}, P_{\sigma} \in \mathbb{R}^{n \times n}, \sigma \in \mathscr{I}$.

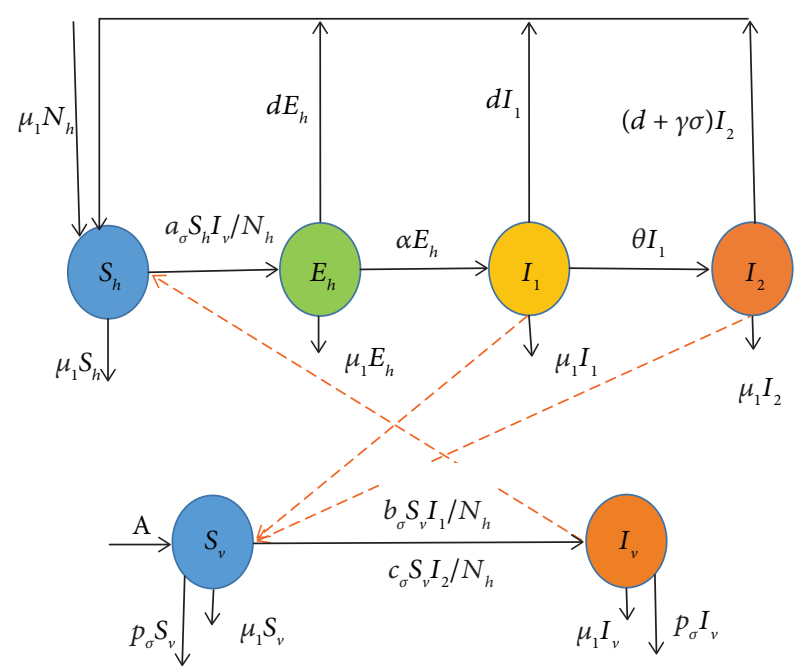

FIgURE 1: A schematic of model (4) showing transitions to different categories for trees and psyllids. Black arrows show the transitions between compartments. Orange dashed arrows show the necessary interactions between trees and psyllids to obtain transmission.

Particularly, if $\sigma \in \mathscr{I}_{p}$, system (5) can be rewritten as follows:

$$
\begin{aligned}
\dot{x}(t) & =A_{k}(t) x(t), \quad t \in\left(t_{k-1}, t_{k}\right], \\
x\left(t_{k}^{+}\right) & =P_{k} x\left(t_{k}\right), \quad t=t_{k}, k \in \mathbb{N}, \\
x\left(t_{0}^{+}\right) & =x_{0}, \quad t_{0} \geq 0,
\end{aligned}
$$

where $A_{k+m}(t)=A_{k}(t), P_{k+m}=P_{k}$, and $t_{k}-t_{k-1}=\omega_{k}$ with $\omega_{k+m}=\omega_{k}$, and then $\omega=\sum_{k=1}^{m} \omega_{k}$ is the period of switch $\sigma$.

Let $\Psi_{A_{k}}(t, s)(t \geq s)$ be the evolution operator of the linear $\omega$-periodic system

$$
\dot{x}(t)=A_{k}(t) x(t), \quad x \in \mathbb{R}^{n} .
$$

Denote

$$
\Phi_{A_{k} P_{k}}(\omega):=\prod_{k=1}^{m}\left(P_{m-k+1} \Psi_{A_{k}}\left(t_{m-k+1}, t_{m-k}\right)\right) .
$$

Lemma 1. If $\eta=(1 / \omega) \ln r\left(\Phi_{A_{k} P_{k}}(\omega)\right)$, then there exists a positive $\omega$-periodic vector function $v(t)$ such that $\exp (\eta t) v(t)$ is a solution of system (6).

Since the proof is similar to that of Lemma 1 in [24], so one omits it.

Lemma 2. If $r\left(\Phi_{A_{k} P_{k}}(\omega)\right)<1$, then the trivial solution of system (6) is asymptotically stable.

Using the similar method in [25], this result can be easily proved (not shown in this paper).

2.2. $R_{0}$ for General Impulsive Periodic System with Switching Parameters. Consider a general impulsive switching system with periodic environment: 


$$
\begin{aligned}
\dot{x}(t) & =f^{k}(x), \quad t \in\left(t_{k-1}, t_{k}\right], \\
x\left(t^{+}\right) & =\psi^{k}(x(t)), \quad t=t_{k}, k \in \mathbb{N}, \\
x\left(t_{0}^{+}\right) & =x_{0}, \quad t_{0} \geq 0,
\end{aligned}
$$

where $f^{k}: \mathbb{R}_{+}^{\mathrm{n}} \rightarrow \mathbb{R}^{n}, \psi^{k}: \mathbb{R}_{+}^{n} \rightarrow \mathbb{R}_{+}^{n}, f^{k+m}=f^{k}$, and $\psi^{k+m}=$ $\psi^{k}$.

Following [26], we split the compartments by two types with the first $q$ compartments $\left\{x_{1}, x_{2}, \ldots, x_{q}\right\}$ the infected individuals and $\left\{x_{q+1}, x_{q+2}, \ldots, x_{n}\right\}$ the uninfected individuals. And denote $X=\left(x_{1}, x_{2}, \ldots, x_{q}\right), Y=\left(x_{q+1}, x_{q+2}\right.$, $\left.\ldots, x_{n}\right), \psi^{k}=\left(h^{k}, g^{k}\right)^{T}, h^{k}=\left(\psi_{1}^{k}, \psi_{2}^{k}, \ldots, \psi_{q}^{k}\right)$, and $g^{k}=\left(\psi_{q+1}^{k}\right.$, $\left.\ldots, \psi_{n}^{k}\right)$. Define

$$
X_{s}=\left\{x \in \mathbb{R}_{+}^{n} \mid x_{i}=0, i=1, \ldots, q\right\} .
$$

We can rewrite system (9) as:

$$
\begin{aligned}
\dot{x}(t) & =\mathscr{F}^{k}(x(t))-\mathscr{V}^{k}(x(t)), \quad t \in\left(t_{k-1}, t_{k}\right], \\
X\left(t^{+}\right) & =h^{k}(x(t)), \\
Y\left(t^{+}\right) & =g^{k}(x(t)), \\
t & =t_{k}, k \in \mathbb{N}, \\
x\left(t^{+}\right) & =x_{0}, \quad t_{0} \geq 0,
\end{aligned}
$$

where $\mathscr{F}^{k}(x)$ are the newly infected rates, $\mathscr{V}^{k+}(x)$ are the input rates of individuals by other means, and $\mathscr{V}^{k-}(x)$ are the rates of transfer of individuals out of compartments; then, $\mathscr{V}^{k}(x)=\mathscr{V}^{k-}(x)-\mathscr{V}^{k+}(x)$ represent the set transfer rates out of compartments. Thus, $f^{k}(x)=\mathscr{F}^{k}(x)-\mathscr{V}^{k}(x)$. We assume that system (11) satisfies $\mathscr{F}^{k+m}=\mathscr{F}^{k}, \mathscr{V}^{k+m}=\mathscr{V}^{k}, h^{k+m}=h^{k}$, and $g^{k+m}=g^{k}$, and system (11) has a disease-free periodic solution $x^{*}(t)$.

We make the following assumptions, which share the same biological meanings as those by Wang and Zhao [27] and Yang and Xiao [28].

(H1) If $x_{i} \geq 0$, then the function $\mathscr{F}_{i}^{k}(x), \mathscr{V}_{i}^{k+}(x)$, and $\mathscr{V}_{i}^{k-}(x)$ are nonnegative and continuous on $\mathbb{R}_{+}^{n}$ and continuously differential with respect to $x$ for $i=1, \ldots, n$.

(H2) If $x_{i}=0$, then $\mathscr{V}_{i}^{k-}(x)=0$. Particularly, if $x \in X_{s}$, then $\mathscr{V}_{i}^{k-}(x)=0$ for $i=1, \ldots, q$.

(H3) $\mathscr{F}_{i}^{k}(x)=0$ for $q+1, \ldots, n$.

(H4) If $x \in X_{s}$, then $\mathscr{F}_{i}^{k}(x)=\mathscr{V}_{i}^{k+}(x)=0$ for $i=1, \ldots, q$.

(H5) The pulse on the infected compartments must be uncoupled with the uninfected compartments; that is, $h^{k}\left(x\left(t_{k}\right)\right)$ is essentially $h^{k}\left(X\left(t_{k}\right)\right)$, and $h^{k}(\mathbf{0})=\mathbf{0}$.

(H6) $r\left(\Phi_{M_{k} Q_{k}}(\omega)\right)<1$, where $\Phi_{M_{k} Q_{k}}(\omega)=\prod_{k=1}^{m}\left(Q_{m-k+1}\right.$ $\left.\Psi_{M_{k}}\left(t_{m-k+1}, t_{m-k}\right)\right)$, and $\Phi_{M_{k} Q_{k}}(t)$ is the fundamental solution matrix of the following system:

$$
\begin{aligned}
\dot{Z}(t) & =M_{k}(t) Z(t), \quad t \in\left(t_{k-1}, t_{k}\right] \\
Z\left(t_{k}^{+}\right) & =Q_{k} Z\left(t_{k}\right), \quad t=t_{k}, k \in \mathbb{N},
\end{aligned}
$$

where

$$
\begin{aligned}
M_{k}(t) & =\left(\frac{\partial f_{i}^{k}\left(x^{*}(t)\right)}{\partial x_{j}}\right)_{q+1 \leq i, j \leq n}, \\
Q_{k} & =\left(\frac{\partial \psi_{i}^{k}\left(x^{*}\left(t_{k}\right)\right)}{\partial x_{j}}\right)_{q+1 \leq i, j \leq n} .
\end{aligned}
$$

From (H2)-(H4), the derivatives of $\mathscr{F}^{k}\left(x^{*}(t)\right)$ and $\mathscr{V}^{k}\left(x^{*}(t)\right)$ can be parted as follows:

$$
\begin{aligned}
D \mathscr{F}^{k}\left(x^{*}(t)\right) & =\left(\begin{array}{cc}
F_{k}(t) & 0 \\
0 & 0
\end{array}\right), \\
D \mathscr{V}^{k}\left(x^{*}(t)\right) & =\left(\begin{array}{cc}
V_{k}(t) & 0 \\
J_{k} & -M_{k}(t)
\end{array}\right),
\end{aligned}
$$

where

$$
\begin{aligned}
& F_{k}(t)=\left(\frac{\partial \mathscr{F}_{i}^{k}\left(x^{*}(t)\right)}{\partial x_{j}}\right)_{1 \leq i, j \leq \mathrm{q}}, \\
& V_{k}(t)=\left(\frac{\partial \mathscr{V}_{i}^{k}\left(x^{*}(t)\right)}{\partial x_{j}}\right)_{1 \leq i, j \leq q} .
\end{aligned}
$$

Furthermore, it follows from (H5) that $h^{k}$ are the functions of $X\left(t_{k}\right)$. So the derivatives of $\psi^{k}\left(x^{*}\left(t_{k}\right)\right)$ can be separated as follows:

$$
D \psi^{k}\left(x^{*}\left(t_{k}\right)\right)=\left(\begin{array}{cc}
P_{k} & 0 \\
\Gamma_{k} & Q_{k}
\end{array}\right),
$$

where $P_{k} \in \mathbb{R}^{q \times q}$ and $\Gamma_{k} \in \mathbb{R}^{(n-q) \times q}$ defined by

$$
\begin{aligned}
& P_{k}=\left(\frac{\partial \psi_{i}^{k}\left(x^{*}\left(t_{k}\right)\right)}{\partial x_{j}}\right)_{1 \leq i, j \leq q}, \\
& \Gamma_{k}=\left(\frac{\partial \psi_{i}^{k}\left(x^{*}\left(t_{k}\right)\right)}{\partial x_{j}}\right)_{q+1 \leq i \leq n, 1 \leq j \leq q} .
\end{aligned}
$$

(H7) $r\left(\Phi_{-V_{k}(t) P_{k}}(\omega)\right)<1$.

In addition, from Assumption (H7) and Lemma 2, we can see that the trivial solution of the following linear switching system with impulses

$$
\begin{gathered}
\dot{y}(t)=-V_{k} y(t), \quad t \in\left(t_{k-1}, t_{k}\right], \\
y\left(t^{+}\right)=P_{k} y(t), \quad t=t_{k}, k \in \mathbb{N},
\end{gathered}
$$


is asymptotically stable. According to Remark 3.5 in Sect. III. 7 of [29], we have that there exist constants $K>0$ and $\rho>0$, such that

$$
\|Y(t, s)\| \leq K \exp \{-\rho(t-s)\}, \quad \forall t \geq s, s \in \mathbb{R},
$$

where $Y(t, s)$ is the evolution operator of system (18).

Similar to the notation and definition of [24], we define the so-called next infection operator $L$,

$$
\begin{aligned}
L \phi(t) & =\int_{-\infty}^{t} Y(t, s) F(s) \phi(s) \mathrm{d} s \\
& =\int_{0}^{\infty} Y(t, t-a) F(t-a) \phi(t-a) d a, \quad \forall t \in \mathbb{R}_{+},
\end{aligned}
$$

where $\phi(s)$ is a $\omega$-periodic function from $\mathbb{R}$ to $\mathbb{R}_{+}^{q}$ and denotes the initial distribution of infections individuals, and $F(t)=F_{k}(t)$ when $t \in\left(t_{k-1}, t_{k}\right]$.

Now, we define the basic reproductive number $\mathscr{R}_{0}$ for system (11) as follows:

$$
\mathscr{R}_{0}=r(L) .
$$

In order to calculate the implicit expression $\mathscr{R}_{0}$ by numerical simulation, we consider the auxiliary $\omega$-periodic switching system with impulses:

$$
\begin{aligned}
\dot{U}(t) & =\left[-V_{k}(t)+\frac{F_{k}(t)}{\lambda}\right] U(t), \quad t \in\left(t_{k-1}, t_{k}\right], \\
U\left(t^{+}\right) & =P_{k} U(t), \quad t=t_{k}, k \in \mathbb{N},
\end{aligned}
$$

where $\lambda \in(0, \infty)$. Set $U(t, s, \lambda)(t \geq s, s \in \mathbb{R})$ to be the evolution operator of system (22), then $U(\omega, 0, \lambda)=\Phi_{\left(\left(F_{k} / \lambda\right)-V_{k}\right) P_{k}}$ $(\omega)$. Following the idea in [28], we have following results.

Lemma 3. Assuming that (H1)-(H7) hold, then the following statements are valid:

(i) If $r\left(\Phi_{\left(\left(F_{k} / \lambda\right)-V_{k}\right) P_{k}}(\omega)\right)=1$ has a positive solution $\lambda_{0}$, then $\lambda_{0}$ is an eigenvalue of $L$, and so $\mathscr{R}_{0}>0$.

(ii) If $\mathscr{R}_{0}>0$, then $\lambda=\mathscr{R}_{0}$ is the unique solution of $r\left(\Phi_{\left(\left(F_{k} / \lambda\right)-V_{k}\right) P_{k}}(\omega)\right)=1$.

(iii) $\mathscr{R}_{0}=0$ if and only if $r\left(\Phi_{\left(\left(F_{k} / \lambda\right)-V_{k}\right) P_{k}}(\omega)\right)<1$ for all $\lambda>0$.

By applying Lemma 3, one knows that $\mathscr{R}_{0}$ for impulsive periodic switching system (11) is the solution of algebraic equation $r\left(\Phi_{\left(\left(F_{k} / \lambda\right)-V_{k}\right) P_{k}}(\omega)\right)=1$.

Lemma 4. Assuming that (H1)-(H7) hold, then the following statements are valid for system (11):

(i) $\mathscr{R}_{0}=1$ if and only if $r\left(\Phi_{\left(F_{k}-V_{k}\right) P_{k}}(\omega)\right)=1$.

(ii) $\mathscr{R}_{0}>1$ if and only if $r\left(\Phi_{\left(F_{k}-V_{k}\right) P_{k}}(\omega)\right)>1$.

(iii) $\mathscr{R}_{0}<1$ if and only if $r\left(\Phi_{\left(F_{k}-V_{k}\right) P_{k}}(\omega)\right)<1$.
It follows from Lemma 4 that the disease-free periodic solution $x^{*}(t)$ of system (11) is asymptotically stable if $\mathscr{R}_{0}<$ 1 and unstable if $\mathscr{R}_{0}>1$.

\section{Main Results}

In this section, we are going to explore the threshold condition which leads to the extinction and persistence of the disease for impulsive switching model (4) for HLB with seasonal fluctuations.

Lemma 5. All solutions of system (4) with nonnegative initial conditions are nonnegative for all $t>t_{0}$ and ultimately bounded.

The proof of Lemma 5 is simple; we omit it.

Referring to [21], we can get that system (4) has a unique disease-free periodic solution $x^{*}(t)=\left(0,0,0,0, S_{h}^{*}(t), S_{v}^{*}(t)\right)$, where $S_{h}^{*}(t)$ and $S_{v}^{*}(t)$ are the unique periodic solution of the following systems, respectively:

$$
\frac{d S_{h}(t)}{d t}=\mu_{1} N_{h}-\mu_{1} S_{h}(t)
$$

and

$$
\begin{aligned}
& \frac{d S_{v}(t)}{d t}=\Lambda-\mu_{2} S_{v}(t), \quad t \neq t_{k}, \\
& S_{v}\left(t^{+}\right)=\left(1-p_{k}\right) S_{v}(t), \quad t=t_{k} .
\end{aligned}
$$

We can easily obtain that Assumptions (H1)-(H5) hold for system (4). Next, we will show that Assumptions (H6) and (H7) hold. By (13), (15), and (17), we can calculate $M_{k}$, $Q_{k}, F_{k}, V_{k}$, and $P_{k}$ of system (4), which are represented as the following form:

$$
\begin{aligned}
M_{k}(t) & =\left(\begin{array}{cc}
-\mu_{1} & 0 \\
0 & -\mu_{2}
\end{array}\right), \\
Q_{k} & =\left(\begin{array}{cc}
1 & 0 \\
0 & 1-p_{k}
\end{array}\right), \\
F_{k}(t) & =\left(\begin{array}{cccc}
0 & 0 & 0 & a_{k} \\
0 & 0 & 0 & 0 \\
0 & 0 & 0 & 0 \\
0 & \frac{c_{k} S_{v}^{*}(t)}{N_{h}} & \frac{b_{k} S_{v}^{*}(t)}{N_{h}} & 0
\end{array}\right),
\end{aligned}
$$

$$
V_{k}(t)=\left(\begin{array}{cccc}
\mu_{1}+\alpha+d & 0 & 0 & 0 \\
-\alpha & \mu_{1}+\theta+d & 0 & 0 \\
0 & -\theta & \mu_{1}+d+\gamma_{k} & 0 \\
0 & 0 & 0 & \mu_{2}
\end{array}\right)
$$




$$
P_{k}=\left(\begin{array}{cccc}
1 & 0 & 0 & 0 \\
0 & 1 & 0 & 0 \\
0 & 0 & 1 & 0 \\
0 & 0 & 0 & 1-p_{k}
\end{array}\right) .
$$

By calculating, we get

$$
\Phi_{M_{k} Q_{k}}(\omega)=\left(\begin{array}{cc}
\exp \left(-\mu_{1} \omega\right) & 0 \\
0 & \prod_{k=1}^{m}\left(1-p_{k}\right) \exp \left(-\mu_{2} \omega\right)
\end{array}\right) \text {, }
$$

and

$$
\Phi_{-V_{k} P_{k}}(\omega)=\left(\begin{array}{cccc}
J_{1} & 0 & 0 & 0 \\
* & J_{2} & 0 & 0 \\
* & * & J_{3} & 0 \\
0 & 0 & 0 & J_{4}
\end{array}\right),
$$

where $J_{1}=\exp \left\{-\left(\mu_{1}+d+\alpha\right) \omega\right\}, J_{2}=\exp \left\{-\left(\mu_{1}+d+\theta\right) \omega\right\}$, $J_{3}=\exp \left\{\sum_{k=1}^{m}-\left(\mu_{1}+d+\gamma_{k}\right) \omega_{k}\right\}, \quad$ and $\quad J_{4}=\prod_{k=1}^{m}\left(1-p_{k}\right)$ $\exp \left(-\mu_{2} \omega\right)$. There is no need to calculate the exact forms of $*$, as they are not required in the analysis that follows. Obviously, $r\left(\Phi_{M_{k} Q_{k}}(\omega)\right)<1$ and $r\left(\Phi_{-V_{k} P_{k}}(\omega)\right)<1$. Thus, Assumptions (H6) and (H7) hold.

Theorem 1. If $\mathscr{R}_{0}<1$, then the disease-free periodic solution $x^{*}(t)$ of system (4) is globally asymptotically stable, whereas it is unstable if $\mathscr{R}_{0}>1$.

Proof 1. From Lemma 4, one has that the unique disease-free periodic solution $x^{*}(t)$ is unstable if $\mathscr{R}_{0}>1$, and $x^{*}(t)$ is locally stable if $\mathscr{R}_{0}<1$. Therefore, one only needs to show the global attractivity of $x^{*}(t)$ for $\mathscr{R}_{0}<1$.

From Lemma 4 , we get $r\left(\Phi_{\left(F_{k}-V_{k}\right) P_{k}}(\omega)\right)<1$ since $\mathscr{R}_{0}<1$. So we can choose a sufficiently small $\varepsilon_{1}>0$ such that

$$
\begin{aligned}
& r\left(\Phi_{\left(F_{k}-V_{k}+M_{\varepsilon_{1} k}\right) P_{k}}(\omega)\right)<1, \\
& \text { where } M_{\varepsilon_{1} k}=\left(\begin{array}{cccc}
0 & 0 & 0 & 0 \\
0 & 0 & 0 & 0 \\
0 & 0 & 0 & 0 \\
0 & \frac{c_{k} \varepsilon_{1}}{N_{h}} & \frac{b_{k} \varepsilon_{1}}{N_{h}} & 0
\end{array}\right) .
\end{aligned}
$$

From system (4), we have that

$$
\begin{gathered}
\frac{d S_{v}(t)}{d t} \leq \Lambda-\mu_{2} S_{v}(t), \quad t \neq t_{k}, \\
S_{v}\left(t^{+}\right)=\left(1-p_{k}\right) S_{v}(t), \quad t=t_{k} .
\end{gathered}
$$

By comparison theorem in impulsive differential equations, for the abovementioned $\varepsilon_{1}$, we have that there exists a $\bar{T}_{1}>0$ such that

$$
S_{v}(t) \leq S_{v}^{*}(t)+\varepsilon_{1}, \quad \text { for } t>\bar{T}_{1} .
$$

According to system (4) and inequality (30), we can get that for $t>\bar{T}_{1}$,

$$
\begin{aligned}
\frac{d E_{h}(t)}{d t} & \leq a_{k} I_{v}-\mu_{1} E_{h}-\alpha E_{h}-d E_{h}, \\
\frac{d I_{1}(t)}{d t} & =\alpha E_{h}-\mu_{1} I_{1}-\theta I_{1}-d I_{1}, \\
\frac{d I_{2}(t)}{d t} & =\theta I_{1}-\mu_{1} I_{2}-d I_{2}-\gamma_{k} I_{2}, \\
\frac{d I_{v}(t)}{d t} & \leq \frac{c_{k}\left(S_{v}^{*}(t)+\varepsilon_{1}\right) I_{1}}{N_{h}}+\frac{b_{k}\left(S_{v}^{*}(t)+\varepsilon_{1}\right) I_{2}}{N_{h}}-\mu_{2} I_{v}, \\
t & \in\left(t_{k-1}, t_{k}\right], \\
E_{h}\left(t^{+}\right) & =E_{h}(t), \\
I_{1}\left(t^{+}\right) & =I_{1}(t), \\
I_{2}\left(t^{+}\right) & =I_{2}(t), \\
I_{v}\left(t^{+}\right) & =\left(1-p_{k}\right) I_{v}(t), \\
t & =t_{k} .
\end{aligned}
$$

Consider the following comparison system:

$$
\begin{aligned}
\frac{d \bar{J}(t)}{d t} & =\left(F_{k}(t)-V_{k}(t)+M_{\varepsilon_{1} k}\right) \bar{J}(t), \quad t \neq t_{k}, \\
\bar{J}\left(t^{+}\right) & =P_{k} \bar{J}(t), \quad t=t_{k},
\end{aligned}
$$

where $\bar{J}(t)=\left(\bar{E}_{h}(t), \bar{I}_{1}(t), \bar{I}_{2}(t), \bar{I}_{v}(t)\right)^{T}$.

In view of Lemma 1 , there exists a positive $\omega$-periodic vector function $v_{1}(t)$ such that $\bar{J}(t)=v_{1}(t) \exp (\varsigma t)$ is a solution of system (32), where $\varsigma=\ln r\left(\Phi_{\left(F_{k}-V_{k}+M_{\varepsilon_{1} k}\right) P_{k}}(\omega)\right)<0$. So $\bar{J}(t) \rightarrow 0$, as $t \rightarrow \infty$. It follows (28) that $\lim _{t \rightarrow \infty} \bar{E}_{h}(t)=0$, $\lim _{t \rightarrow \infty} \bar{I}_{1}(t)=0, \lim _{t \rightarrow \infty} \bar{I}_{2}(t)=0$, and $\lim _{t \rightarrow \infty} \bar{I}_{v}(t)=0$. By the comparison theorem in impulsive differential equations, we have $\lim _{t \rightarrow \infty} E_{h}(t)=0, \lim _{t \rightarrow \infty} I_{1}(t)=0, \lim _{t \rightarrow \infty} I_{2}(t)=0$, and $\lim _{t \rightarrow \infty} I_{v}(t)=0$. By the theory of asymptotic semiflows, we can get

$$
\begin{aligned}
& \lim _{t \rightarrow \infty} S_{h}(t)=S_{h}^{*}(t), \\
& \lim _{t \rightarrow \infty} S_{v}(t)=S_{v}^{*}(t) .
\end{aligned}
$$

Hence, the disease-free periodic solution $x^{*}(t)$ is globally asymptotically stable.

Theorem 2. If $\mathscr{R}_{0}>1$, then the disease is uniformly persistent for system (4); that is, there is a positive constant $\epsilon>0$, such that $\lim \inf _{t \rightarrow \infty} E_{h}(t)>\epsilon, \lim \inf _{t \rightarrow \infty} I_{1}(t)>\epsilon, \lim \inf _{t \rightarrow \infty} I_{2}$ $(t)>\epsilon$, and $\lim \inf _{t \rightarrow \infty} I_{v}(t)>\epsilon$. 
Proof 2. Denote $\tilde{K}=\left\{\left(E_{h}, I_{1}, I_{2}, I_{v}, S_{h}, S_{v}\right) \in \mathbb{R}_{+}^{6}\right\}, K_{0}=\left\{\left(E_{h}\right.\right.$, $\left.I_{1}, I_{2}, I_{v}, S_{h}, S_{v}\right) \in \tilde{K}: E_{h}>0, I_{1}>0, I_{2}>0, I_{v}>0, S_{h} \geq 0, S_{v} \geq$ $0\}$, and $\partial K_{0}=\tilde{K} \backslash K_{0}$. Let $u\left(t, t_{0}, x_{0}\right)$ be the unique solution of system (4) with the initial value $x_{0}=\left(E_{h}^{0}, I_{1}^{0}, I_{2}^{0}, I_{v}^{0}, S_{h}^{0}, S_{v}^{0}\right)$ at time $t_{0}$.

Define Poincaré map $P: \tilde{K} \rightarrow \tilde{K}$ associated with system (4) as follows:

$$
P\left(x_{0}\right)=u\left(t_{0}+\omega^{+}, x_{0}\right), \quad \forall x_{0} \in \tilde{K}, \forall \mathrm{t}_{0} \in \mathbb{R}_{+} .
$$

Set

$$
\begin{aligned}
M_{\partial}=\{ & \left(E_{h}^{0}, I_{1}^{0}, I_{2}^{0}, I_{v}^{0}, S_{h}^{0}, S_{v}^{0}\right) \\
& \in \partial K_{0} \mid P^{m}\left(E_{h}^{0}, I_{1}^{0}, I_{2}^{0}, I_{v}^{0}, S_{h}^{0}, S_{v}^{0}\right) \\
& \left.\in \partial K_{0}, \forall m \in \mathbb{Z}_{+}\right\} .
\end{aligned}
$$

One claims that

$$
M_{\partial}=\left\{\left(0,0,0,0, S_{h}, S_{v}\right) \mid S_{h} \geq 0, S_{v} \geq 0\right\} .
$$

Obviously, $\left\{\left(0,0,0,0, S_{h}, S_{v}\right) \mid S_{h} \geq 0, S_{v} \geq 0\right\} \subseteq M_{\partial}$. Next, one wants to show

$$
M_{\partial} \backslash\left\{\left(0,0,0,0, S_{h}, S_{v}\right) \mid S_{h} \geq 0, S_{v} \geq 0\right\}=\varnothing .
$$

If (37) does not hold, then there exists a point $\left(E_{h}^{0}, I_{1}^{0}, I_{2}^{0}\right.$, $\left.I_{v}^{0}, S_{h}^{0}, S_{v}^{0}\right) \in M_{\partial} \backslash\left\{\left(0,0,0,0, S_{h}, S_{v}\right) \mid S_{h} \geq 0, S_{v} \geq 0\right\}$. Next, for four initial values $E_{h}^{0}, I_{1}^{0}, I_{2}^{0}$, and $I_{v}^{0}$, three cases should be discussed.

Case (i). One initial value equals zero, and the others are larger than zero. Without loss of generality, one chooses $E_{h}^{0}=0, I_{1}^{0}>0, I_{2}^{0}>0$, and $I_{v}^{0}$ $>0$. It is obvious that $S_{h}(t)>0$ and $I_{v}(t)>0$ for any $t \geq t_{0}$. Then, from the first equation of system (4), one gets $\left.\left(d E_{h}(t) / d t\right)\right|_{t=t_{0}}=$ $a_{1} S_{h}\left(t_{0}\right) I_{v}\left(t_{0}\right) / N_{h}>0$. Thus, $\left(E_{h}, I_{1}, I_{2}, I_{v}\right.$, $\left.S_{h}, S_{v}\right) \notin \partial K_{0}$ for $0<t-t_{0} \ll 1$. This is a contradiction. Other cases are similarly proved.

Case (ii). Two initial values equal zero, and the others are larger than zero. One lets $E_{h}^{0}=I_{1}^{0}=0, I_{2}^{0}>$ 0 , and $I_{v}^{0}>0$. It is obvious that $S_{h}(t)>0$ and $I_{v}(t)>0$ for any $t \geq t_{0}$. Using the same method as aforementioned, one can prove $\left(E_{h}, I_{1}\right.$, $\left.I_{2}, I_{v}, S_{h}, S_{v}\right) \notin \partial K_{0}$ for $0<t-t_{0} \ll 1$. This is a contradiction. Other cases can be proved similarly.

Case (iii). Three initial values equal zero, and the other is larger than zero. Set $E_{h}^{0}=I_{2}^{0}=I_{v}^{0}=0$ and $I_{1}^{0}>0$. It is obvious that $S_{v}(t)>0$ and $I_{1}(t)>0$ for any $t \geq t_{0}$. Then, from the fourth equation of system (4), one gets $\left.\left(d I_{v}(t) / d t\right)\right|_{t=t_{0}}=b_{1} S_{v}\left(t_{0}\right) I_{1}$ $\left(t_{0}\right) / N_{h}>0$. Thus, $\left(E_{h}, \mathrm{I}_{1}, I_{2}, I_{v}, S_{h}, S_{v}\right) \notin \partial K_{0}$ for $0<t-t_{0} \ll 1$. This is a contradiction. Similarly, one can prove the other cases.
Thus,

$$
M_{\partial}=\left\{\left(0,0,0,0, S_{h}, S_{v}\right) \mid S_{h} \geq 0, S_{v} \geq 0\right\} .
$$

In the following, one proceeds by contradiction to prove that there exists $\widehat{\xi}>0$ such that

$\limsup _{t \rightarrow \infty} d\left(P^{m}\left(E_{h}^{0}, I_{1}^{0}, I_{2}^{0}, I_{v}^{0}, S_{h}^{0}, S_{v}^{0}\right), P_{0}\right) \geq \widehat{\xi}, \quad \forall x_{0} \in \tilde{K}, m \in \mathbb{Z}_{+}$.

where $P_{0}=\left(0,0,0,0, S_{h}^{*}\left(t_{0}\right), S_{v}^{*}\left(t_{0}\right)\right)$.

By Lemma 4, one has $r\left(\Phi_{\left(F_{k}-V_{k}\right) P_{k}}(\omega)\right)>1$ if $\mathscr{R}_{0}>1$. So one can choose $\varepsilon_{2}>0$ sufficiently small such that

$$
\begin{aligned}
r\left(\Phi_{\left(F_{k}-V_{k}-M_{\varepsilon_{2} k}\right) P_{k}}(\omega)\right) & >1, \\
\text { where } M_{\varepsilon_{2} k}= & \left(\begin{array}{cccc}
0 & 0 & 0 & \frac{a_{k} \varepsilon_{2}}{N_{h}} \\
0 & 0 & 0 & 0 \\
0 & 0 & 0 & 0 \\
0 & \frac{c_{k} \varepsilon_{2}}{N_{h}} & \frac{b_{k} \varepsilon_{2}}{N_{h}} & 0
\end{array}\right) .
\end{aligned}
$$

If (39) does not hold, then for any $\widehat{\xi}>0$, one obtains

$$
\begin{array}{r}
\limsup _{t \rightarrow \infty} d\left(P^{m}\left(E_{h}^{0}, I_{1}^{0}, I_{2}^{0}, I_{v}^{0}, S_{h}^{0}, S_{v}^{0}\right), P_{0}\right)<\widehat{\xi}, \\
\quad \text { for some }\left(E_{h}^{0}, I_{1}^{0}, I_{2}^{0}, I_{v}^{0}, S_{h}^{0}, S_{v}^{0}\right) \in \tilde{K} .
\end{array}
$$

Without loss of generality, one supposes that

$$
d\left(P^{m}\left(E_{h}^{0}, I_{1}^{0}, I_{2}^{0}, I_{v}^{0}, S_{h}^{0}, S_{v}^{0}\right), P_{0}\right)<\widehat{\xi}, \quad \forall \widehat{\xi}>0, m \in \mathbb{Z}_{+} .
$$

By the continuity of the solution with respect to initial values, one has that there exists sufficiently small $\widehat{\xi}$ such that

$$
\begin{array}{r}
\left\|u\left(t, P^{m}\left(E_{h}^{0}, I_{1}^{0}, I_{2}^{0}, I_{v}^{0}, S_{h}^{0}, S_{v}^{0}\right)\right)-u\left(t, P_{0}\right)\right\| \leq \varepsilon_{2}, \\
\forall t \in\left[t_{0}, t_{0}+\omega\right], \forall m \in \mathbb{Z}_{+} .
\end{array}
$$

For any $t \geq t_{0}$, there exists an integer $l \in \mathbb{Z}_{+}$such that $t=l \omega+\widehat{t}$, where $\hat{t} \in\left[t_{0}, t_{0}+\omega\right]$. Then one has

$$
\begin{aligned}
& \left\|u\left(t, P^{m}\left(E_{h}^{0}, I_{1}^{0}, I_{2}^{0}, I_{v}^{0}, S_{h}^{0}, S_{v}^{0}\right)\right)-u\left(t, P_{0}\right)\right\| \\
& \quad=\left\|u\left(\hat{t}, P^{m}\left(E_{h}^{0}, I_{1}^{0}, I_{2}^{0}, I_{v}^{0}, S_{h}^{0}, S_{v}^{0}\right)\right)-u\left(\widehat{t}, P_{0}\right)\right\| \leq \varepsilon_{2} .
\end{aligned}
$$

Therefore, one has

$$
\begin{array}{r}
S_{h}(t) \geq S_{h}^{*}(t)-\varepsilon_{2}, \\
S_{v}(t) \geq S_{v}^{*}(t)-\varepsilon_{2}, \\
\quad \text { for all } t \geq t_{0} .
\end{array}
$$

From system (4) and inequality (45), one gets 


$$
\begin{aligned}
\frac{d E_{h}(t)}{d t} & \geq \frac{a_{k}\left(S_{h}^{*}(t)-\varepsilon_{2}\right) I_{v}}{N_{h}}-\mu_{1} E_{h}-\alpha E_{h}-d E_{h}, \\
\frac{d I_{1}(t)}{d t} & =\alpha E_{h}-\mu_{1} I_{1}-\theta I_{1}-d I_{1}, \\
\frac{d I_{2}(t)}{d t} & =\theta I_{1}-\mu_{1} I_{2}-d I_{2}-\gamma_{k} I_{2}, \\
\frac{d I_{v}(t)}{d t} & \geq \frac{c_{k}\left(S_{v}^{*}(t)-\varepsilon_{2}\right) I_{1}}{N_{h}}+\frac{b_{k}\left(S_{v}^{*}(t)-\varepsilon_{2}\right) I_{2}}{N_{h}}-\mu_{2} I_{v}, \\
t & \in\left(t_{k-1}, t_{k}\right], \\
E_{h}\left(t^{+}\right) & =E_{h}(t), \\
I_{1}\left(t^{+}\right) & =I_{1}(t), \\
I_{2}\left(t^{+}\right) & =I_{2}(t), \\
I_{v}\left(t^{+}\right) & =\left(1-p_{k}\right) I_{v}(t), \\
t & =t_{k}, k \in \mathbb{N} .
\end{aligned}
$$

Consider the comparison system for system (46):

$$
\begin{aligned}
\frac{d Z(t)}{d t} & =\left(F_{k}(t)-V_{k}(t)-M_{\varepsilon_{2} k}\right) Z(t), \quad t \neq t_{k} \\
Z\left(t^{+}\right) & =P_{k} Z(t), \quad t=t_{k}
\end{aligned}
$$

where $Z(t)=\left(E_{h}(t), I_{1}(t), I_{2}(t), I_{v}(t)\right)^{T}$.

By Lemma 1, one knows that there exists a positive $\omega$-periodic vector function $v_{2}(t)$ such that $Z(t)=v_{2}(t)$ $\exp (\zeta t)$ is a solution of system (47), where $\zeta=\ln r$ $\left(\Phi_{\left(F_{k}-V_{k}-M_{\varepsilon_{2}}\right) P_{k}}(\omega)\right)$. From (40), one can get that $Z(t)$ $\rightarrow \infty$ as $t \rightarrow \infty$, and $E_{h}(t) \rightarrow \infty, I_{1}(t) \rightarrow \infty, I_{2}(t) \rightarrow \infty$, and $I_{v}(t) \rightarrow \infty$ as $t \rightarrow \infty$. By the comparison theorem in impulsive differential equations, one has $E_{h}(t) \rightarrow \infty$, $I_{1}(t) \rightarrow \infty, \quad I_{2}(t) \rightarrow \infty$, and $I_{v}(t) \rightarrow \infty$ as $t \rightarrow \infty$. This contradicts with the boundedness of the solutions. Thus, one has proved that (39) holds and $P$ is weakly uniformly persistent with respect to $\left(K_{0}, \partial K_{0}\right)$.

Obviously, the Poincaré map $P$ has a global attractor $P_{0}$. $P_{0}$ is an isolated invariant set in $\tilde{K}$ and $W^{s}\left(P_{0}\right) \cap K_{0}=\varnothing$ and it is acyclic in $M_{\partial}$. Every solution in $M_{\partial}$ converges to $P_{0}$. According to Zhao [30], one derives that $P$ is uniformly persistent with respect to $\left(K_{0}, \partial K_{0}\right)$. This implies that the solution of system (4) is uniformly persistent with respect to $\left(K_{0}, \partial K_{0}\right)$. This completes the proof.

\section{Numerical Simulations}

In this section, we first provide results from numerical simulations of model (4) that demonstrate and support our theoretical results. For these simulations, part of parameters values for model (4) are outlined in Table 1.

In [20], Zhao revealed that 1-2 sprays should be done in the period after picking and before spring sprout, in spring, summer growth, and in autumn growth. So we assume that the system is composed of four subsystems, and the switching law is periodic and satisfies
TABLE 1: Parameter values for system (4).

\begin{tabular}{lccc}
\hline Parameter & Value & Unit & Reference \\
\hline$\Lambda$ & $6,028,433$ & year $^{-1}$ & {$[31]$} \\
$N_{h}$ & 2000 & - & {$[31]$} \\
$\mu_{1}$ & 0.04 & year $^{-1}$ & {$[32]$} \\
$\mu_{2}$ & 3.2 & year $^{-1}$ & {$[33]$} \\
$d$ & 0.025 & year $^{-1}$ & {$[21]$} \\
$\alpha$ & 24.33 & year $^{-1}$ & {$[15]$} \\
$\theta$ & 1.8 & year $^{-1}$ & {$[16,17]$} \\
$\omega$ & 1 & year $^{-1}$ & - \\
$k$ & 0.6 & - & Estimation \\
\hline
\end{tabular}
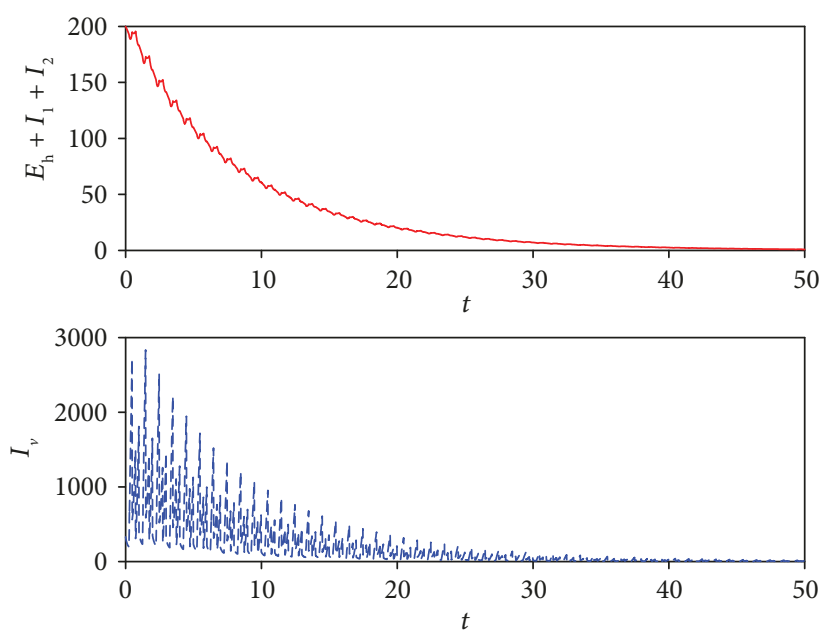

FIgURE 2: Time series plots of the total of infected trees and infected psyllids. The disease dies out with $\mathscr{R}_{0}=0.903702<1$.

$$
\sigma(t)=\left\{\begin{aligned}
& 1, \text { if } t \in(k, k+0.25], \text { winter, } \\
& 2, \text { if } t \in(k+0.25, k+0.5], \text { spring, } \\
& 3, \text { if } t \in(k+0.5, k+0.75], \text { summer, } \\
& 4, \text { if } t \in(k+0.75, k+1], \text { autumn, } \\
& k=0,1,2, \ldots
\end{aligned}\right.
$$

Consider dynamical behavior of system (4) with initial conditions $E_{h}^{0}=100, I_{1}^{0}=40, I_{2}^{0}=60, I_{v}^{0}=330, S_{h}^{0}=1800$, and $S_{v}^{0}=10028$. The switched parameter values are used as follows: $a_{1}=0.002, a_{2}=0.078, a_{3}=0.05, a_{4}=0.078, b_{1}=$ $0.006, b_{2}=0.156, b_{3}=0.106$, and $b_{4}=0.156$. For the control switched parameter values, we set $\gamma_{1}=0.2, \gamma_{2}=0.8, \gamma_{3}=$ $0.2, \gamma_{4}=0.8, p_{1}=0, p_{2}=0.8, p_{3}=0.8$, and $p_{4}=0.8$. According to Lemma 3 , we can get $\mathscr{R}_{0}=0.903702<1$ by numerical calculation, which shows that the disease dies out (see Figure 2). Set $\gamma_{1}=0.2, \gamma_{2}=0.4, \gamma_{3}=0.2, \gamma_{4}=0.35, p_{1}=0$, $p_{2}=0.2, p_{3}=0.15$, and $p_{4}=0.5$. We get $\mathscr{R}_{0}=1.801682>1$; the disease is uniformly persistent by Theorem 2 , which is showed from Figure 3. 

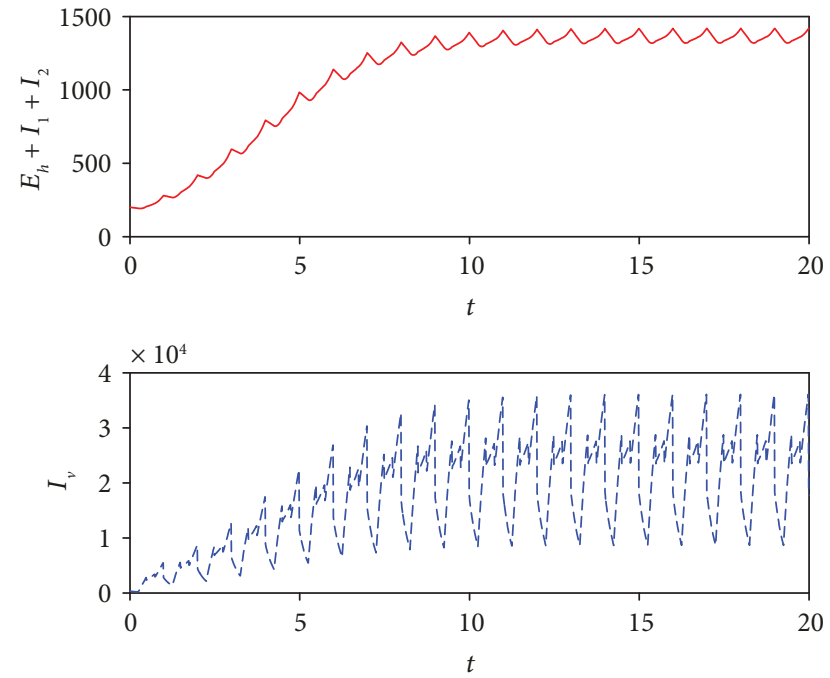

FIGURE 3: Time series plots of the total of infected trees and infected psyllids. The disease is persistent with $\mathscr{R}_{0}=1.801682>1$.

TABle 2: The effect of parameters $p_{\sigma}$ and $\gamma_{\sigma}$ on the disease control.

\begin{tabular}{lccccccccc}
\hline & $p_{1}$ & $p_{2}$ & $p_{3}$ & $p_{4}$ & $\gamma_{1}$ & $\gamma_{2}$ & $\gamma_{3}$ & $\gamma_{4}$ & $\mathscr{R}_{0}$ \\
\hline Baseline scenario & 0.3 & 0.8 & 0.3 & 0.8 & 0.2 & 0.8 & 0.2 & 0.8 & 0.878143 \\
Strategy I & 0.3 & 0.8 & 0.3 & 0.8 & 0.8 & 0.2 & 0.8 & 0.2 & 0.868525 \\
Strategy II & 0.8 & 0.3 & 0.8 & 0.3 & 0.2 & 0.8 & 0.2 & 0.8 & 0.994301 \\
\hline
\end{tabular}

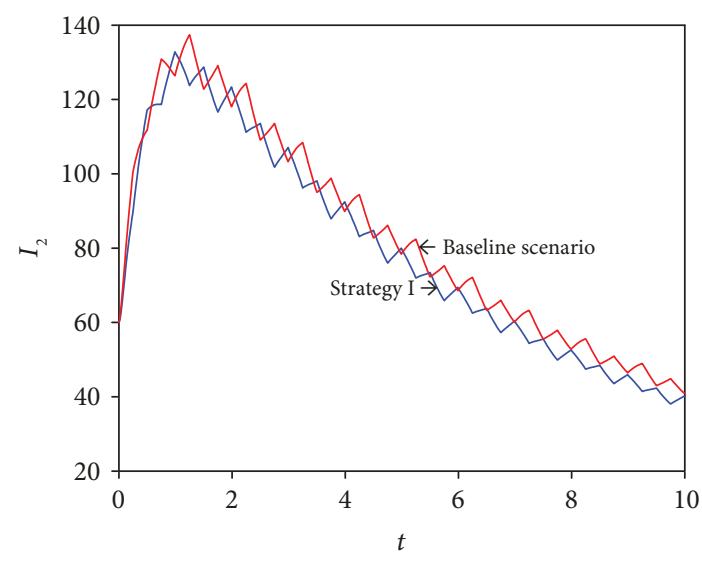

Figure 4: Time series plot of the total of infected trees for the baseline scenario and Strategy I.

Switching parameters have an effect on the peak size of infected individuals for switching epidemic models. Next, we consider the effect of varying switching removal rates $\gamma_{\sigma}$ and insecticide spraying rates $p_{\sigma}$ to evaluate the effectiveness of various control measures, while holding the other switched parameters constant. In Table 2, we give two different control projects to compare with the baseline scenario, which is denoted by Strategy I and Strategy II.

Figures 4 and 5 show the numerical simulations of the baseline scenario, Strategy I, and Strategy II. If we compare the baseline scenario and Strategy I (see rows 1 and 2), the evaluation implies that the baseline scenario is worse than

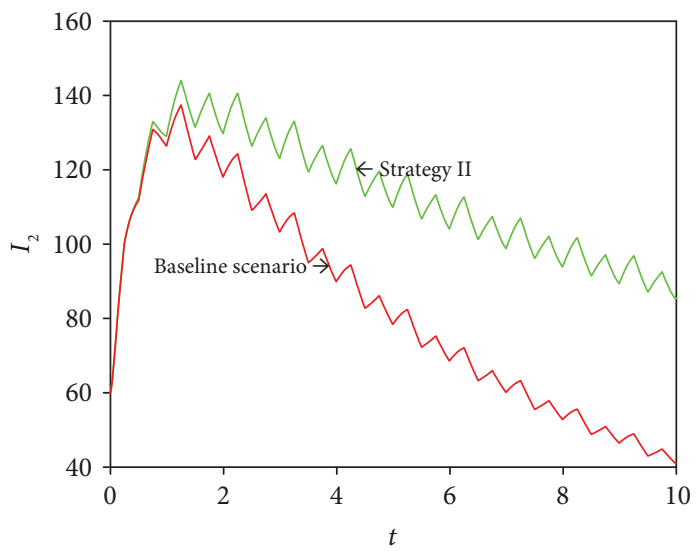

FIgURE 5: Time series plot of the total infected trees for the baseline scenario and Strategy II.

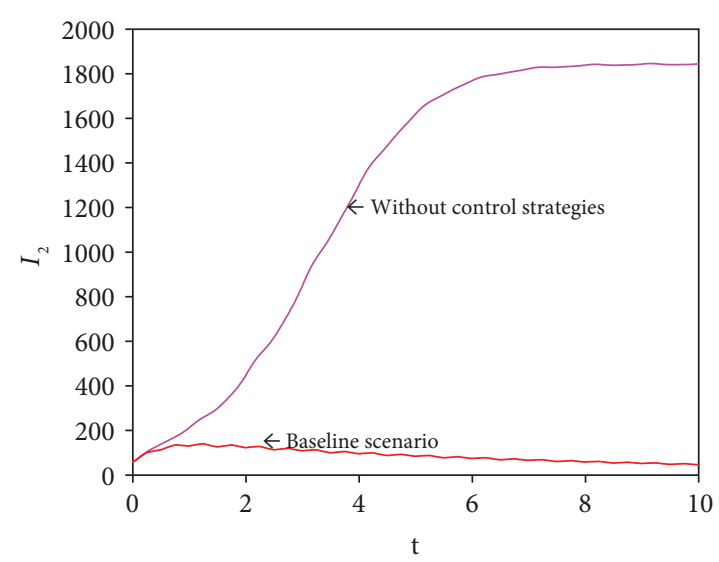

FIgURE 6: Time series plot of the total infected trees for the baseline scenario and without control strategies.

Strategy I (larger final and peak sizes and $\mathscr{R}_{0}$ ). If we compare the baseline scenario and Strategy II (see rows 1 and 3), the evaluation suggests that the baseline scenario is better than Strategy II (lower final and peak sizes and $\mathscr{R}_{0}$ ). This illustrates that Strategy I is the best control project, and the most effective control strategy is spraying in spring and autumn and removing in winter.

By calculating, $\mathscr{R}_{0}=5.023193$ in the absence of control strategies. We can observe from Figure 6 that the disease breaks out rapidly. This illustrates that removing infected trees and spraying pesticides play an important role in controlling the spread of HLB.

\section{Conclusions}

By introducing switching parameters into a general impulsive HLB model, a novel impulsive switching model for HLB with seasonal fluctuations has been constructed and a threshold value $\mathscr{R}_{0}$ with switching effect has been established to measure whether the disease is uniformly persistent. The modeling and analytic methods presented in this paper improve the classical results for the systems with impulsive 
interventions. Numerical examples have been given to demonstrate the effectiveness of the results obtained.

Our numerical investigations demonstrate that the most effective season of spraying insecticide is in spring and autumn and the most effective season of removing infected trees is winter. The result strongly suggests and supports the previous observations $[19,34]$. This can serve as an integrating measure to design an appropriate strategy to control HLB spread.

\section{Conflicts of Interest}

The authors declare that there are no conflicts of interest regarding the publication of this paper.

\section{Acknowledgments}

The research has been supported by the National Natural Science Foundation of China (11561004) and the Natural Science Foundation of Jiangxi Province (20171BAB201006).

\section{References}

[1] M. Nageswara-Rao, M. Irey, S. M. Garnsey, and S. Gowda, "Candidate gene makers for Candidatus, Liberibacter asiaticus for detecting citrus greening disease," Journal of Biosciences, vol. 38, no. 2, pp. 229-237, 2013.

[2] B. Aubert, "Trioza erytreae Del Guercio and Diaphorina citri Kuwayana (Homoptera Psylloidea), the two vectors of citrus greening disease: biological aspects and possible control strategies," Fruits, vol. 42, no. 3, pp. 149-162, 1987.

[3] J. M. Bové and M. E. Rogers, "Huanglongbing-control workshop: summary," vol. 1065, Acta Horticulturae, 2015.

[4] A. J. Ayres, J. Belasque Jr., and J. M. Bové, “The experience with huanglongbing management in Brazil," Acta Horticulturae, vol. 1065, pp. 55-61, 2015.

[5] G. Fan, B. Liu, R. Wu, T. Li, Z. Cai, and C. Ke, "Thirty years of research on citrus huanglongbing in China," Fujian Journal of Agricultural Sciences, vol. 24, no. 2, pp. 183-190, 2009.

[6] K. Jacobsen, J. Stupiansky, and S. S. Pilyugin, "Mathematical modeling of citrus groves infected by huanglongbing," Mathematical Biosciences and Engineering, vol. 10, no. 3, pp. 705728, 2013.

[7] G. MacDonald, "The dynamics of helminth infections, with special reference to schistosomes," Transactions of the Royal Society of Tropical Medicine and Hygiene, vol. 59, no. 5, pp. 489-506, 1965.

[8] A. D. Barbour, "Macdonald's model and the transmission of bilharzia," Transactions of the Royal Society of Tropical Medicine and Hygiene, vol. 72, no. 1, pp. 6-15, 1978.

[9] H.-M. Wei, X.-Z. Li, and M. Martcheva, "An epidemic model of a vector-borne disease with direct transmission and time delay," Journal of Mathematical Analysis and Applications, vol. 342, no. 2, pp. 895-908, 2008.

[10] A. Abdelrazec, J. Bélair, C. Shan, and H. Zhu, "Modeling the spread and control of dengue with limited public health resources," Mathematical Biosciences, vol. 271, pp. 136-145, 2016.

[11] A. Abdelrazec, S. Lenhart, and H. Zhu, "Transmission dynamics of West Nile virus in mosquitoes and corvids and non- corvids," Journal of Mathematical Biology, vol. 68, no. 6, pp. 1553-1582, 2014.

[12] C. Chiyaka, B. H. Singer, S. E. Halbert, J. G. Morris Jr, and A. H. C. van Bruggen, "Modeling huanglongbing transmission within a citrus tree," Proceedings of the National Academy of Sciences of the United States of America, vol. 109, no. 30, pp. 12213-12218, 2012.

[13] R. M. Anderson and R. M. May, "Helminth infections of humans: mathematical models, population dynamics, and control," Advances in Parasitology, vol. 24, pp. 1-101, 1985.

[14] Y. Yang and D. Xiao, "A mathematical model with delays for schistosomiasis japonicum transmission," Chinese Annals of Mathematics, Series B, vol. 31, no. 4, pp. 433-446, 2010.

[15] J. A. Lee, S. E. Halbert, W. O. Dawson, C. J. Robertson, J. E. Keesling, and B. H. Singer, "Asymptomatic spread of huanglongbing and implications for disease control," Proceedings of the National Academy of Sciences of the United States of America, vol. 112, no. 24, pp. 7605-7610, 2015.

[16] W. Shen, S. E. Halbert, E. Dickstein, K. L. Manjunath, M. M. Shimwela, and A. H. C. van Bruggen, "Occurrence and ingrove distribution of citrus huanglongbing in north central Florida," Journal of Plant Pathology, vol. 95, no. 2, pp. 361371, 2013.

[17] K. L. Manjunath, S. E. Halbert, C. Ramadugu, S. Webb, and R. F. Lee, "Detection of 'Candidatus Liberibacter asiaticus' in Diaphorina citri and its importance in the management of citrus huanglongbing in Florida," Phytopathology, vol. 98, no. 4, pp. 387-396, 2008.

[18] R. G. d.' A. Vilamiu, S. Ternes, G. A. Braga, and F. F. Laranjeira, "A model for huanglongbing spread between citrus plants including delay times and human intervention," in 2012 International Conference of Numerical Analysis and Applied Mathematics. AIP Conference Proceedings, Volume 1479, pp. 2315-2319, Kos, Greece, 2012.

[19] R. A. Taylor, E. A. Mordecai, C. A. Gilligan, J. R. Rohr, and L. R. Johnson, "Mathematical models are a powerful method to understand and control the spread of huanglongbing," PeerJ, vol. 4, p. e2642, 2016.

[20] X. Zhao, Background, Current Situation and Management of the HLB and Its Vector in China, In Memoria del $2^{\circ}$. Taller Intl. sobre Huanglongbing de los cítricos (Candidatus Liberibacter spp) y el psilido asiático de los cítricos (Diaphorina citri), 2nd International Workshop on itrus Huanglongbing and The Asian Citrus Psyllid, Hermosillo, Sonora, Mexico, 2010.

[21] L. Zhang, S. Gao, D. Xie, and F. Zhang, "Varying pulse control schemes for citrus huanglongbing epidemic model with general incidence," Communications in Mathematical Biology and Neuroscience, vol. 2016, no. 7, pp. 1-13, 2016.

[22] M. S. Irey, T. Gast, and T. R. Gottwald, "Comparison of visual assessment and polymerase chain reaction assay testing to estimate the incidence of the huanglongbing pathogen in commercial Florida citrus," Proceedings of the Florida State Horticultural Society, vol. 119, pp. 89-93, 2006.

[23] X. Wang, X. Liu, W. Xie, W. Xu, and Y. Xu, "Global stability and persistence of HIV models with switching parameters and pulse control," Mathematics and Computers in Simulation, vol. 123, no. C, pp. 53-67, 2016.

[24] S. Gao, L. Xia, Y. Liu, and D. Xie, “A plant virus disease model with periodic environment and pulse roguing," Studies in Applied Mathematics, vol. 136, no. 4, pp. 357-381, 2016. 
[25] A. M. Samoilenko and N. A. Perestyuk, Differential equations with impulsive effect, Visca Skola, Kiev, 1987.

[26] P. van den Driessche and J. Watmough, "Reproduction numbers and sub-threshold endemic equilibria for compartmental models of disease transmission," Mathematical Biosciences, vol. 180, no. 1-2, pp. 29-48, 2002.

[27] W. Wang and X. Q. Zhao, "Threshold dynamics for compartmental epidemic models in periodic environments," Journal of Dynamics and Differential Equations, vol. 20, no. 3, pp. 699717, 2008.

[28] Y. Yang and Y. Xiao, "Threshold dynamics for compartmental epidemic models with impulses," Nonlinear Analysis: Real World Applications, vol. 13, no. 1, pp. 224-234, 2012.

[29] D. Bainov and P. Simeonov, Impulsive Differential Equations: Periodic Solutions and Applications, Longman Scientific and Technical Group Limited, New York, NY, USA, 1993.

[30] X. Zhao, Dynamical Systems in Population Biology, Springer, Berlin, NY, USA, 2003.

[31] L. Luo, S. Gao, Y. Ge, and Y. Luo, "Transmission dynamics of a huanglongbing model with cross protection," Advances in Difference Equations, vol. 2017, no. 1, 376 pages, 2017.

[32] M. Deng, "Forming process and basis and technological points of the theory emphasis on control citrus psylla for integrated control huanglongbing," Chinese Agricultural Science Bulletin, vol. 25, pp. 358-363, 2009.

[33] X. Deng and G. Chen, "The newly process of huanglongbing in citrus," Guangxi Horticulture, vol. 17, no. 3, pp. 49-51, 2006.

[34] M. Deng, G. Chen, M. Tang, and T. Chen, "The investigation on the annual population dynamics of Diaphorina Citri Kuwayama and correlation analysis about incidence rate of huanglongbing of next year in the normal orchards," Chinese Agricultural Science Bulletin, vol. 25, no. 18, pp. 360-363, 2009. 


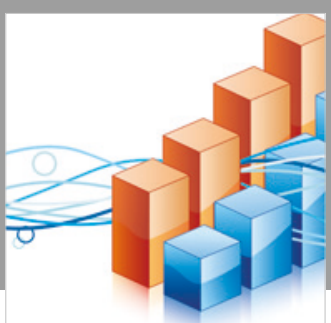

Advances in

Operations Research

\section{-n-m}
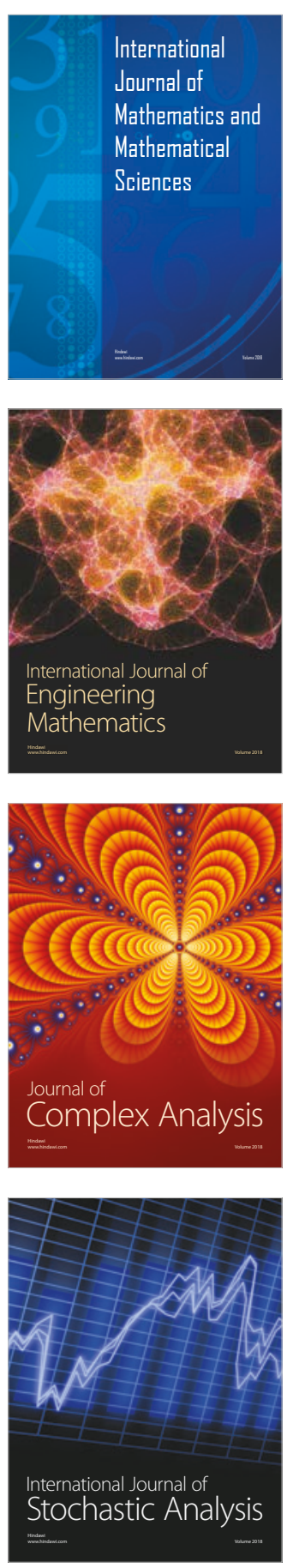
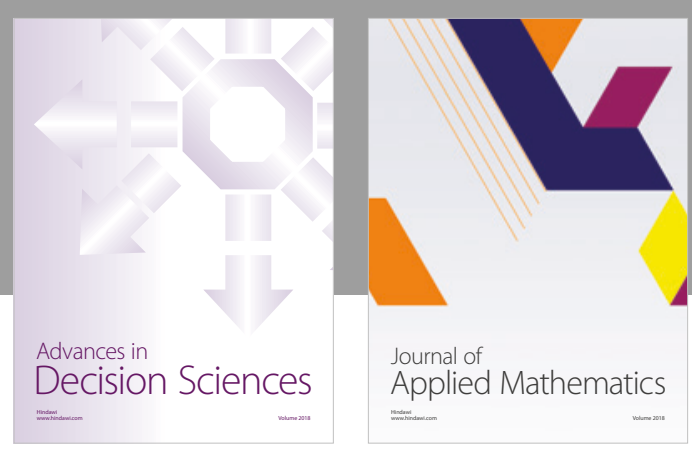

Journal of

Applied Mathematics
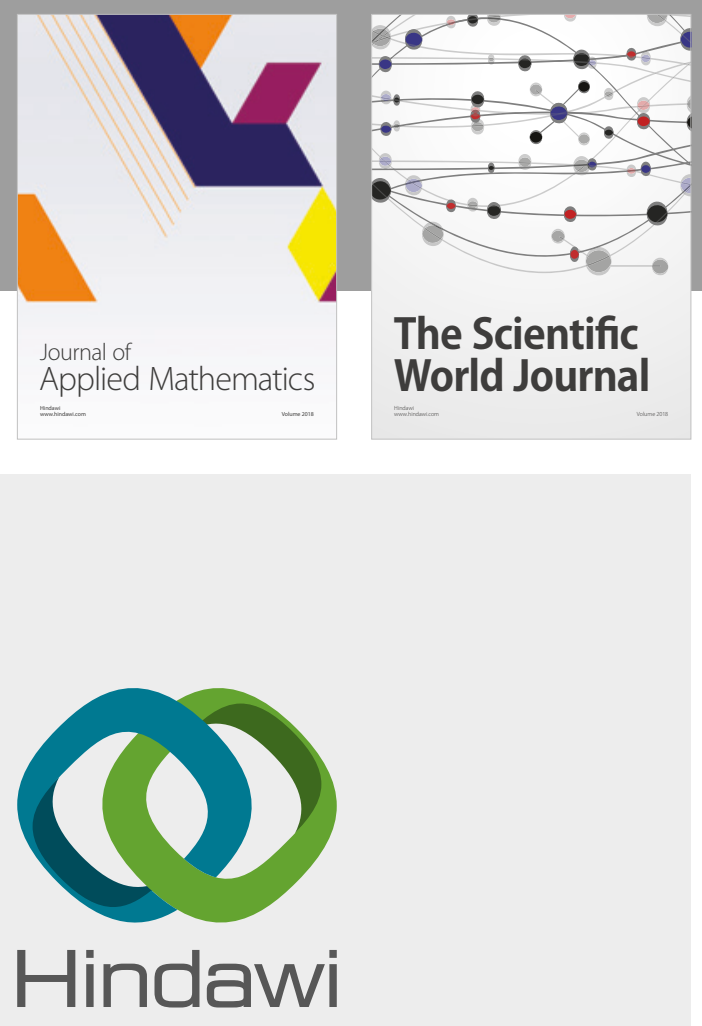

Submit your manuscripts at

www.hindawi.com

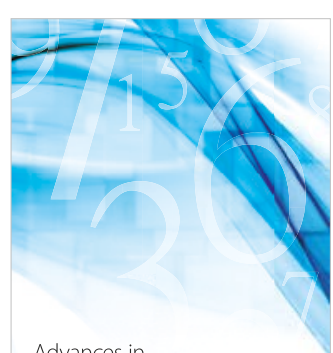

Advances in
Numerical Analysis
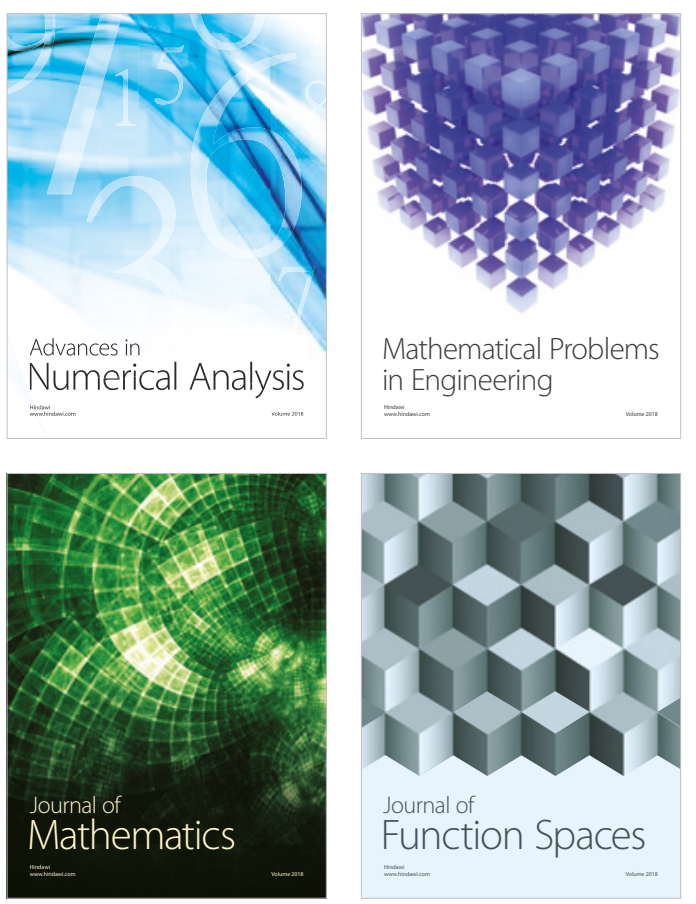

Mathematical Problems in Engineering

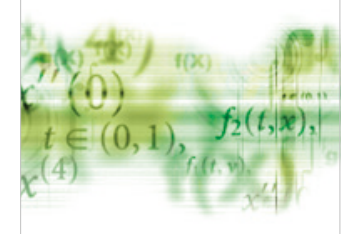

International Journal of

Differential Equations

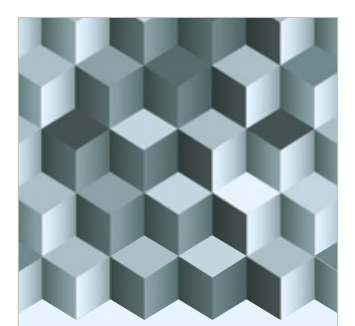

Journal of

Function Spaces
The Scientific

World Journal

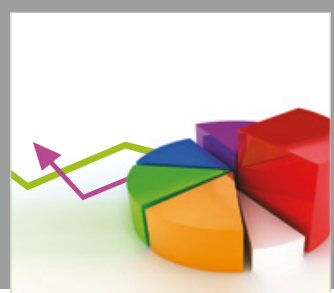

Journal of

Probability and Statistics
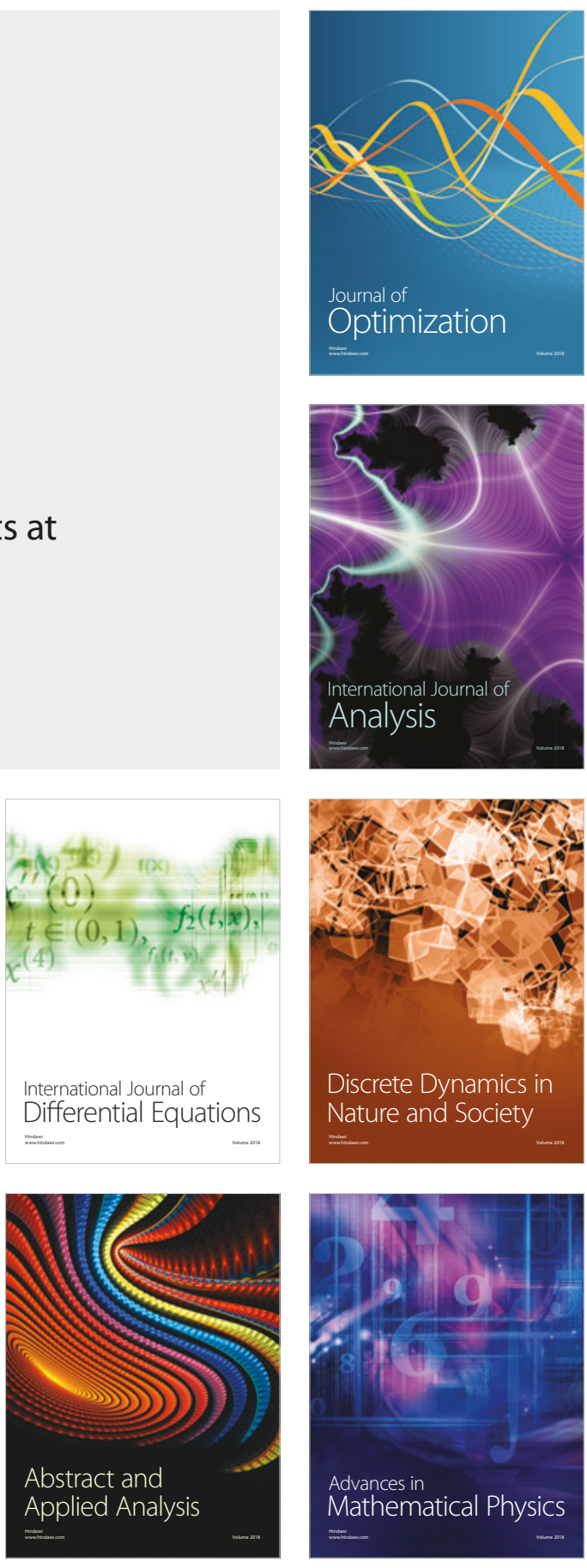